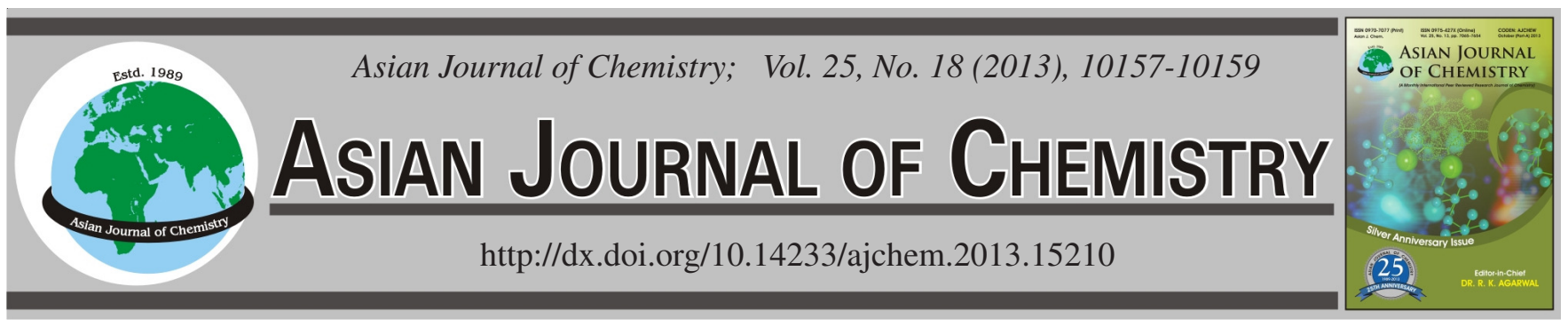

\title{
Micronization and Dissolubility of Griseofulvin Using Rapid Expansion of Supercritical Solution with Cosolvent Process
}

\author{
GuOQIn $\mathrm{Hu}^{*}$, SuJuan Zhang and Banghai XIE
}

School of Chemical Engineering and Energy, Zhengzhou University, Zhengzhou, Henan Province, P.R. China

*Corresponding author: Tel/Fax: +86 371 67781712; E-mail: huguoqin@zzu.edu.cn

Micronization of griseofulvin was prepared using rapid expansion of supercritical solution (RESS) with cosolvent process. The average diameter of precipitated by rapid expansion of supercritical solution was $1 \mu \mathrm{m}$ or so. The results of SEM and DSC showed that the microparticles in crystal habit were not modified evidently at the experimental conditions tested. The dissolving rate of precipitated particles was enhanced in the buffer solution.

Key Words: Micronization, Rapid expansion of supercritical solution, Cosolvent, Dissolubility, Griseofulvin.

ᄂ - - - - - - - - - - - - - - - - - - - - - - - - - -1

\section{INTRODUCTION}

Particle size plays an important role in ensuring high performance in processes involving organic and inorganic particulate materials. Generally, particles with small size and narrow distribution (i.e., large surface area per unit volume) are desirable in many chemical processes such as adsorption, reaction and catalysis. The disadvantages of using the conventional techniques are thermal and chemical degradation of products, a large amount of solvent used and associated disposal problems, broad particle size distribution and solvent residues $^{1,2}$.

The rapid expansion of supercritical solutions (RESS), was recently developed for micronization of particles. In a RESS process, solutes are dissolved in a supercritical fluid, resulting in a solute-laden supercritical phase. By reducing the pressure with an expansion device, fine particles with a narrow size distribution can be obtained.

Griseofulvin (GF) is a pharmaceutical with a powerful antifungal action. It is not applied widely because of its lower bioavailability and higher toxicity. In this study the RESS is employed to micronize griseofulvin. The supercritical fluid is carbon dioxide with cosolvent.

\section{EXPERIMENTAL}

Griseofulvin (purity $99.2 \%$ ) was supplied by Shanghai Chinese Pharmaceutical Factory. It consisted of particles ranging from about 2.0-18 $\mu \mathrm{m} . \mathrm{CO}_{2}$ (purity $99.8 \%$ ) was purchased from Shanghai Nanhui Chemical Company and acetone (analytic purity $>99.5 \%$ ) from Shanghai Feida Company.
Choice of cosolvent: Reverchon et al. ${ }^{3}$ used $\mathrm{CHF}_{3}$ as supercritical solvent to micronize griseofulvin. However, its cost is very high. $\mathrm{Chen}^{4}$ used $\mathrm{CO}_{2}$ as supercritical solvent, but the solubility of griseofulvin in supercritical $\mathrm{CO}_{2}$ is very low. In this study, the RESS with cosolvent is employed to micronize griseofulvin.

In this work, acetone was used as cosolvent because it has low boiling point and can increase solubility of griseofulvin in supercritical $\mathrm{CO}_{2}$. The experimental apparatus used for RESS with cosolvent is shown in reference ${ }^{5}$.

Characterization of particles: The crystallinity and melting point of the original material and precipitates were tested by differential scanning calorimetry ( DSC, Universal V2.3C TA Instrument ). The feature of particles precipitated by RESS was analyzed by scanning electron microscope (SEM, Cambridge, British, S-250MK3). Particle size distribution (PSD) was subsequently determined by counting at least 150 particles from the optical microscope images.

Standard curve of griseofulvin: The standard buffer solution of potassium dihydrogen phosphate-disodium hydrogen phosphate ( $\mathrm{pH} 7.4$ ) was prepared to simulate dissolubility of griseofulvin in the environment of body fluid. The absorbance of griseofulvin in the buffer solution with different concentrations was measured by UV-3000. The experimental data between absorbance and concentration of griseofulvin were correlated in Fig. 1. It showed that the relationship between concentration y and absorbance $\mathrm{A}$ is linear from 0-40 $\mathrm{mg} / \mathrm{L}$, its standard curve equation is $\mathrm{y}=49.9024 \mathrm{~A}-0.07345$; $\mathrm{R}=99.98 \%$. 


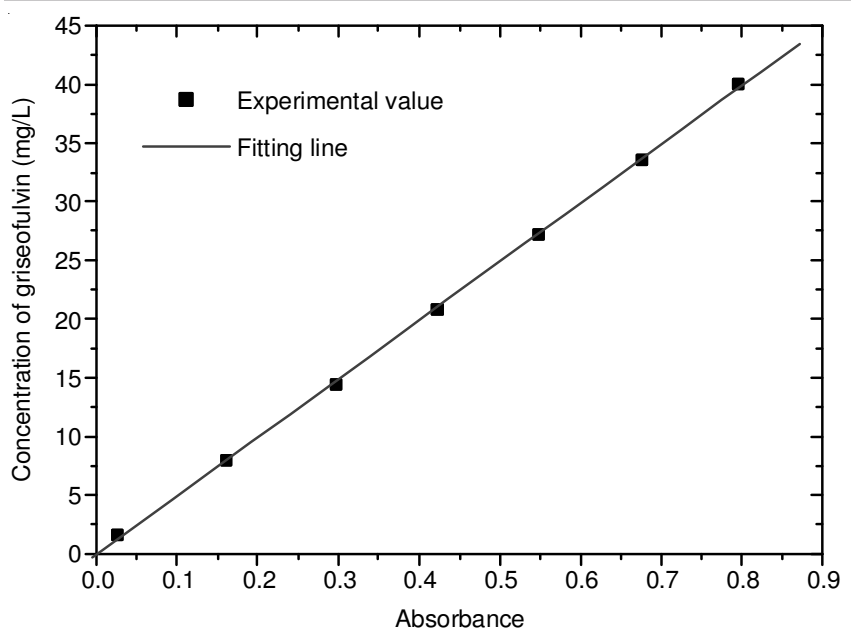

Fig. 1. Standard curve of griseofulvin

\section{RESULTS AND DISCUSSION}

The experimental conditions carried out and results in this study were shown in Table-1. The effects of thermodynamic (e.g., extraction temperature, extraction pressure) and operating (e.g., Spraying distance in expansion chamber, nozzle diameter, cosolvent flowing speed and pre-expansion temperature) conditions on the size and feature of precipitated griseofulvin particles via the RESS had been examined.

Effect of sample spraying distance: When the effect of the spraying distance on precipitates was studied, the other parameters were kept constant. Spraying distances of 6, 13, 20 and $27 \mathrm{~cm}$ measured from the tip of the nozzle to the surface of the stub were used. The results showed that increasing spraying distance caused a decrease in particle size. This may be due to the fact that as the distance increases, the jet has more time to break up and forms discrete particles. Other researchers also found that crystal networking of precipitated salicylic acid decreased as the spraying distance increased ${ }^{6,7}$.
Effect of nozzle diameter: The effect of nozzle diameter on precipitates was investigated for sizes from 20 to $60 \mu \mathrm{m}$ when other parameters were kept constant. The average diameter of the particles precipitated by RESS was $1 \mu \mathrm{m}$ or so. An increase of the nozzle diameter caused an increase in particle size. According to literature ${ }^{8}$ after supercritical solution jets from the exit of nozzle, the supersaturation of the solute increases promptly, so that the generation and growth of crystal nuclei can be finished between the exit of nozzle and Mach disk. Under the same condition of supersaturation the smaller the nozzle diameter is, the larger the jetting velocity is, the faster the growth of crystal nuclei. Therefore, an increase of the nozzle diameter causes an increase in particle size. This result is in agreement with literature ${ }^{9}$.

Effect of cosolvent flowing speed: The effect of cosolvent acetone flowing speed can be evaluated from the results in Table-1. As cosolvent acetone flowing speed increases, the average size decreases from 1.03 to $0.84 \mu \mathrm{m}$, but when the acetone flowing speed excesses $1.2 \mathrm{~mL} / \mathrm{min}$, the acetone can result in being liquid, so we must control the concentration of acetone in this study. It was found that a decrease of the solubility of griseofulvin in supercritical fluid induces a marked increase of particle size due to lower supersaturation and decrease of nucleation rate. Because an increase of concentration of cosolvent acetone caused an increase of the solubility and nucleation rates of griseofulvin in supercritical mixed fluid, the average size decreased.

Effect of extraction temperature: When extraction parameters were studied, the other parameters such as preexpansion temperature, nozzle temperature, diameter of nozzle and temperature of expansion chamber were maintained constant. Table- 1 showed that the extraction temperature has slight effect on the precipitates. An increase of extraction temperature results in a slight decrease in particle size. This may be due to the fact that when the extraction temperature

\begin{tabular}{|c|c|c|c|c|c|c|c|c|}
\hline \multicolumn{9}{|c|}{$\begin{array}{c}\text { TABLE-1 } \\
\text { EXPERIMENTAL CONDITIONS AND RESULTS FOR RESS PROCESSES }\end{array}$} \\
\hline Run No. & $\begin{array}{c}\mathrm{T}_{\text {extraction }} \\
(\mathrm{K})\end{array}$ & $\begin{array}{l}\mathrm{P}_{\text {extraction }} \\
\text { (MPa) }\end{array}$ & $\begin{array}{l}\mathrm{T}_{\text {pre-expansion }} \\
(\mathrm{K})\end{array}$ & $\begin{array}{l}\mathrm{P}_{\text {pre-expasion }} \\
(\mathrm{MPa})\end{array}$ & $\begin{array}{c}\text { Nozzle } \\
\text { diameter }(\mu \mathrm{m})\end{array}$ & $\begin{array}{l}\text { Cosolvent flowing } \\
\text { speed (mL/min) }\end{array}$ & $\begin{array}{c}\text { Spraying } \\
\text { distance }(\mathrm{cm})\end{array}$ & $\begin{array}{l}\text { Average particle } \\
\text { size }(\mu \mathrm{m})\end{array}$ \\
\hline Original & - & - & - & - & - & - & - & 15.0 \\
\hline \multicolumn{9}{|c|}{ Increasing spraying distance } \\
\hline 1 & 323.15 & 24 & 373.15 & 24 & 50 & 0.8 & 6 & 1.20 \\
\hline 2 & 323.15 & 24 & 373.15 & 24 & 50 & 0.8 & 13 & 1.06 \\
\hline 3 & 323.15 & 24 & 373.15 & 24 & 50 & 0.8 & 20 & 1.00 \\
\hline 4 & 323.15 & 24 & 373.15 & 24 & 50 & 0.8 & 27 & 0.86 \\
\hline \multicolumn{9}{|c|}{ Increasing nozzle diameter } \\
\hline 5 & 323.15 & 24 & 373.15 & 24 & 20 & 0.8 & 24 & 0.88 \\
\hline 6 & 323.15 & 24 & 373.15 & 24 & 30 & 0.8 & 24 & 0.90 \\
\hline 7 & 323.15 & 24 & 373.15 & 24 & 40 & 0.8 & 24 & 0.92 \\
\hline 8 & 323.15 & 24 & 373.15 & 24 & 60 & 0.8 & 24 & 1.10 \\
\hline \multicolumn{9}{|c|}{ Increasing cosolvent flowing speed } \\
\hline 9 & 323.15 & 24 & 373.15 & 24 & 50 & 0.4 & 24 & 1.03 \\
\hline 10 & 323.15 & 24 & 373.15 & 24 & 50 & 0.6 & 24 & 0.98 \\
\hline 11 & 323.15 & 24 & 373.15 & 24 & 50 & 1.0 & 24 & 0.84 \\
\hline 12 & 323.15 & 24 & 373.15 & 24 & 50 & 1.2 & 24 & Liquid \\
\hline \multicolumn{9}{|c|}{ Increasing extraction temperature } \\
\hline 13 & 313.15 & 24 & 373.15 & 24 & 50 & 0.8 & 24 & 1.03 \\
\hline 14 & 323.15 & 24 & 373.15 & 24 & 50 & 0.8 & 20 & 0.99 \\
\hline 15 & 333.15 & 24 & 373.15 & 24 & 50 & 0.8 & 24 & 0.98 \\
\hline
\end{tabular}

*Nozzle length is $0.35 \mathrm{~mm}$. 
increases, the solubility of griseofulvin in supercritical $\mathrm{CO}_{2}$ with cosolvent acetone increases. And then the supersaturation of griseofulvin under the same pre-expansion condition is enhanced.

Characterization of particles: The process parameters for characterization of particles are extraction temperature 333 $\mathrm{K}$, pre-expansion pressure $24 \mathrm{MPa}$, pre-expansion temperature $393 \mathrm{~K}$, diameter of nozzle $50 \mu \mathrm{m}$, expansion chamber temperature $303 \mathrm{~K}$ and acetone flowing speed $1.0 \mathrm{~mL} / \mathrm{min}$.

Fig. 2 showed the SEM photographs and PSD of griseofulvin, Fig. $2 \mathrm{a}_{1}$ and $2 \mathrm{~b}_{1}$ are SEM photographs and Fig. $2 \mathrm{a}_{2}$ and $2 b_{2}$ are PSD of original and processed by RESS, respectively. According to SEM photographs and PSD, original average diameter is $8 \mu \mathrm{m}$ or so, but the size processed by RESS is only $1 \mu \mathrm{m}$ and all particles size is less than $2 \mu \mathrm{m}$. Reverchon et al. ${ }^{3}$ observed two different morphologies of griseofulvin crystals under their operating conditions: quasispherical particles and needles. In this study we only found quasispherical particles. The particles obtained in this study were equivalent to that in RESS experiment of Reverchon et al. ${ }^{3}$. As for PSD, the particles size distribution processed by RESS is the narrowest in three methods.

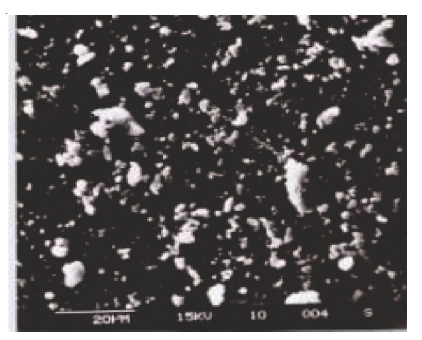

$\left(a_{1}\right)$ SEM of original griseofulvin

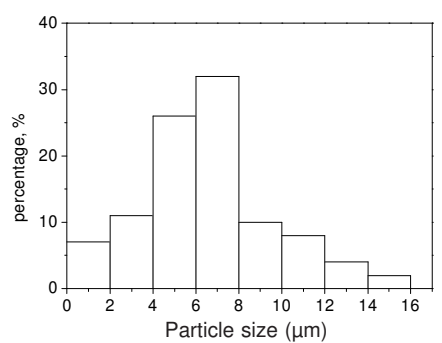

$\left(a_{2}\right)$ PSD of original griseofulvin

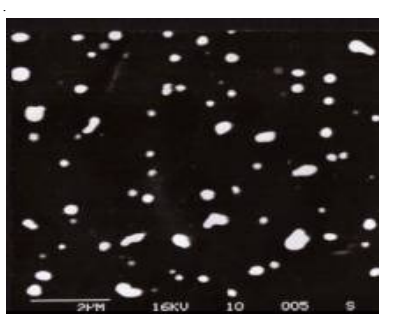

(b $)$ SEM of griseofulvin processed by RESS

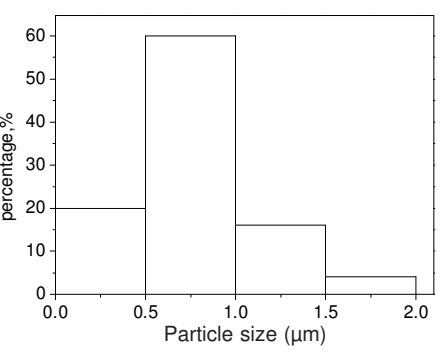

$\left(b_{2}\right)$ PSD of griseofulvin processed by RESS
Fig. 2. SEM photographs and PSD of griseofulvin

Fig. 3 showed heat flow with temperature plot of unprocessed and RESS processed griseofulvin. DSC was performed for thermal analysis of RESS processed and unprocessed griseofulvin particles. Here, DSC analysis was performed for qualitative explanation of the crystallinity change and not so much for quantitative analysis. The melting point of unprocessed griseofulvin is $221.31{ }^{\circ} \mathrm{C}$ whereas after processing it is $218.46^{\circ} \mathrm{C}$. There is no significant change in melting point which suggests that particle crystal form does not change after RESS.

Dissolubility in buffer solution: Fig. 4 showed the dissolubility variation with time for processed by mechanism and RESS griseofulvin particles. The dissolved rates of griseofulvin precipitates processed by RESS much bigger than that of

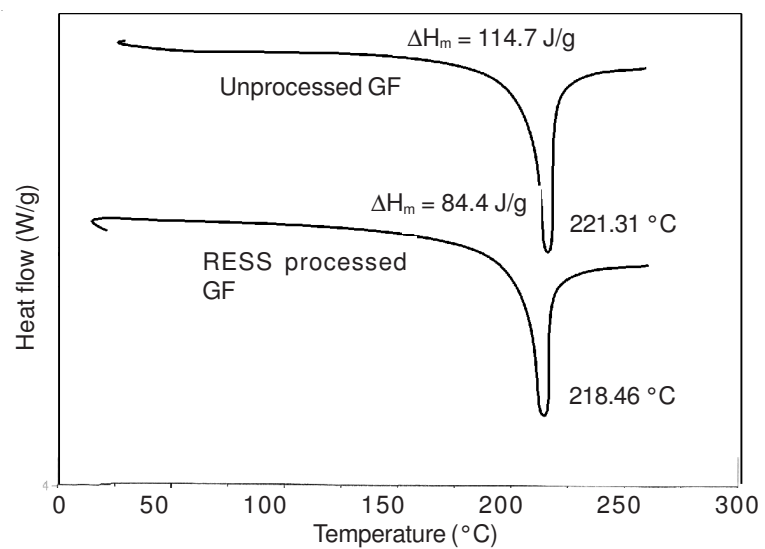

Fig. 3. DSC of RESS processed and unprocessed griseofulvin particles

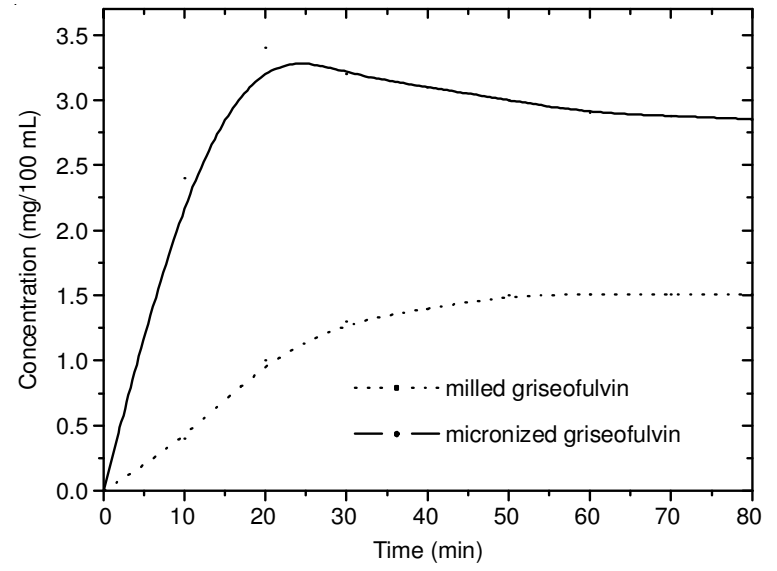

Fig. 4. Dissolubility experiment of griseofulvin

processed by mechanism and reached maximum after $20 \mathrm{~min}$. The result is very important for griseofulvin to be used in clinic.

\section{Conclusion}

Micronization of griseofulvin was successfully performed by RESS using supercritical $\mathrm{CO}_{2}$ with cosolvent acetone. Increasing the spraying distance, pre-expansion pressure cosolvent flowing speed and extraction temperature reduced the particle size. Increasing nozzle diameter and increased the particle size. The smaller the nozzle diameter and pre-expansion temperature is the smaller the particle processed by RESS. The precipitates were analyzed for crystallinity using DSC. Only slight modification in the crystal habit was found under the experimental conditions. The result of dissolubility of griseofulvin in buffer solution showed the smaller the particle size is the bigger the dissolved rate.

\section{REFERENCES}

1. M. Türk and R. Lietzow, J. Supercrit. Fluids, 45, 346 (2008).

2. M. Türk, J. Supercrit. Fluids, 47, 537 (2009).

3. E. Reverchon, G. Della Porta, R. Taddeo, P. Pallado and A. Stassi, Ind. Eng. Chem. Res., 34, 4087 (1995).

4. H. Chen, Dissertation of Ph.D. East China University of Science and Technology (1999).

5. G.Q. Hu, H.Y. Chen, J.G. Cai and D. Xiu, Chin. J. Chem. Eng., 11, 403 (2003).

6. M. Charoenchaitrakool, F. Dehghani, N.R. Foster and H.K. Chan, Ind. Eng. Chem. Res., 39, 4794 (2000).

7. C. Domingo, E.M. Berends and G.M. Van Rosmalen, J. Cryst. Growth, 166, 989 (1996).

8. P.G. Debenedetti, AIChE J., 36, 1289 (1990).

9. A.Z. Hezave and F. Esmaeilzadeh, J. Supercrit. Fluids, 52, 84 (2010). 